\title{
Measurement of Scattering Rate and Minimum Conductivity in Graphene
}

\author{
Y.-W. Tan ${ }^{1 \dagger}$, Y. Zhang ${ }^{1 \dagger}$, K. Bolotin ${ }^{1}$, Y. Zhao ${ }^{1}$, S. Adam ${ }^{2}$, \\ E. H. Hwang 2 , S. Das Sarma ${ }^{2}$, H. L. Stormer ${ }^{1,3,4}$, and P. Kim ${ }^{1}$ \\ ${ }^{1}$ Department of Physics, Columbia University, New York, New York 10027 \\ ${ }^{2}$ Condensed Matter Theory Center, Department of Physics, \\ University of Maryland, College Park, Maryland 20742 \\ ${ }^{3}$ Department of Applied Physics, Columbia University, New York, New York 10027 and \\ ${ }^{4}$ Bell Labs, Alcatel-Lucent Technologies, Murray Hill, NJ 07974
}

\begin{abstract}
The conductivity of graphene samples with various levels of disorder is investigated for a set of specimens with mobility in the range of $1-20 \times 10^{3} \mathrm{~cm}^{2} / \mathrm{V}$ sec. Comparing the experimental data with the theoretical transport calculations based on charged impurity scattering, we estimate that the impurity concentration in the samples varies from $2-15 \times 10^{11} \mathrm{~cm}^{-2}$. In the low carrier density limit, the conductivity exhibits values in the range of $2-12 e^{2} / h$, which can be related to the residual density induced by the inhomogeneous charge distribution in the samples. The shape of the conductivity curves indicates that high mobility samples contain some short range disorder whereas low mobility samples are dominated by long range scatterers.
\end{abstract}

The recent discovery of graphene [1], a single atomic sheet of graphite, has created an intense research effort on this new material. The uniqueness of the electronic band structure of graphene is exemplified by the charge neutral Dirac points located at the Brillouin zone corner. The linear dispersion relation of the bands crossing these Dirac point can be interpreted in analogy to relativistic fermions of vanishing mass. The nontrivial Berry phase associated with the graphene band structure accounts for unusual half integer shifted quantum Hall effect [2, 3] in the presence of a magnetic field. This chiral nature of the Dirac fermions is also expected to induce nontrivial charge transport phenomena, such as a minimum conductivity [2, 4, 5, 6, 7] and weak anti-localization [8, 9, 10, 11]. Indeed, transport measurements indicate the existence of such a minimum conductivity [2, 4] and the suppression of localization [12] in some graphene samples. Despite qualitative agreement, however, these experimental observations do not agree quantitatively with the theoretical expectations [2, 3, 44]. In order to understand these and other low energy transport phenomena in graphene, it is essential to study relevant carrier scattering mechanisms.

In this Letter, we report low magnetic field transport measurements on 19 graphene devices with various levels of disorder. We infer the scattering mechanisms using the density dependence of the mean free paths and phase coherence lengths and comparing with the corresponding transport theory. The sample mean free path is extracted from the conductivity measurements, and we find that at high carrier density, two different scattering mechanisms determine the density-dependent conductivity.

The graphene pieces used in this work are extracted from bulk graphite crystals and deposited onto $\mathrm{SiO}_{2} / \mathrm{Si}$ substrate using the mechanical method described in previous work [13]. Typically, graphene pieces of lateral size 3-20 $\mu \mathrm{m}$ are chosen for device fabrication. For a few large flake samples (lateral size $>20 \mu \mathrm{m}$ ), we explic-

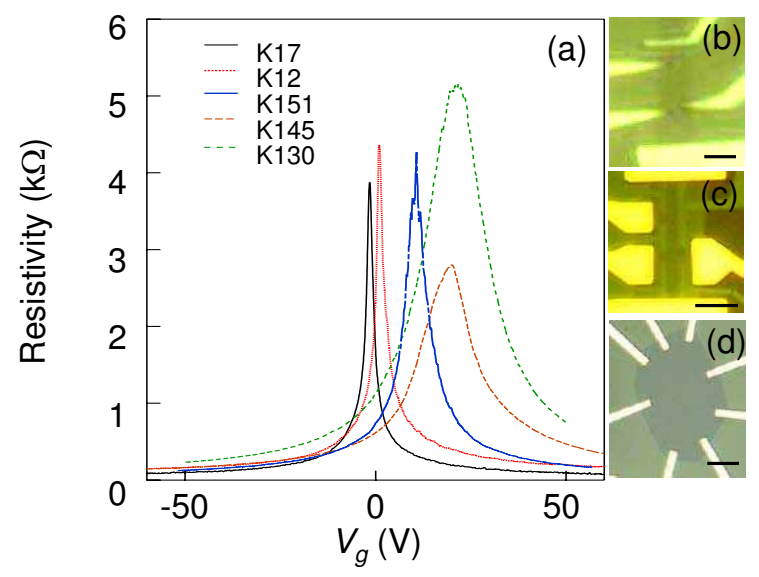

FIG. 1: (color online) (a) Resistivity of four representative graphene samples as a function of applied gate voltage. All data were measured at temperature $1.6 \mathrm{~K}$. (b-d) Optical microscope images of typical devices. The scale bar represents, 1 , and $10 \mu \mathrm{m}$, for (b) and (c, d), respectively

itly fabricated well-defined two to three Hall-bar geometry devices having the same width but varying lengths. These multiple devices from the same graphene piece provide means to check the consistency of our conductivity measurements. The metallic electrodes are defined on the sample using electron beam lithography followed by $\mathrm{Au} / \mathrm{Cr}(30 / 3 \mathrm{~nm})$ evaporation and a lift-off process. The degenerately doped silicon substrate serves as a gate electrode with $300 \mathrm{~nm}$ thermally grown silicon oxide acting as the gate dielectric. By applying a gate bias voltage, $V_{g}$, the charge density of the sample can be tuned. The conductivity of a sample is measured either in the Hall bar geometry considering the width and length of the samples in between the voltage probes (Fig. 1(b)) or by van der Pauw method for samples with irregular shape (Fig. 1(c)). We also use Hall-bar shaped devices (Fig. 1(d)) in order to minimize systematic measurement 


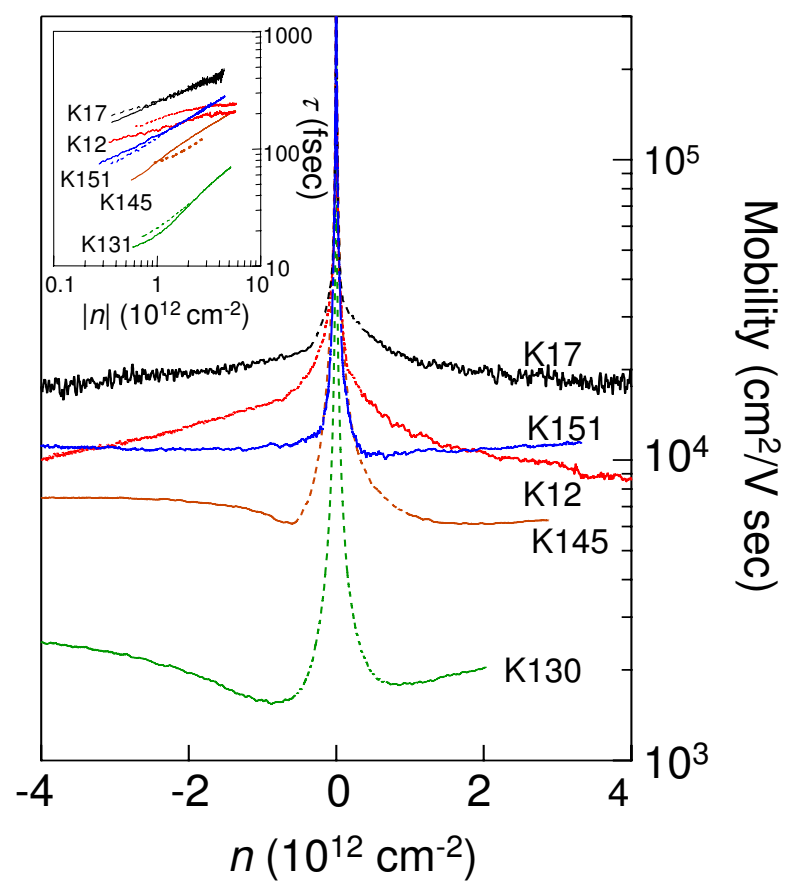

FIG. 2: (color online) Mobility estimated by applying Drude model to the data in Fig. 1. The graphs shown by broken lines are the region where simple Drude model fails to hold. The inset shows the scattering time in this model. The solid lines are for electrons and dashed lines are for holes.

errors. All samples are measured using lock-in amplifiers at an excitation current less than $50 \mathrm{nA}$ to minimize any heating effect on the samples, which are kept in the helium vapor of a cryostat.

Fig. 1(a) shows the resistivities, $\rho$, of five representative samples as a function of $V_{g}$. The resistivity curves are largely symmetric around a particular gate voltage $V_{g}=V_{\text {Dirac }}$ and show a maximum at this value. The finite value of $V_{\text {Dirac }}$ indicates that there exists an unintentional doping of the graphene samples [14] whose origin may be electrostatic or caused by charged impurities. The actual carrier density $n$ in graphene induced by the gate voltage in the presence of impurity doping is then obtained from $n=C_{g}\left(V_{g}-V_{\text {Dirac }}\right) / e$ where the gate capacitance $C_{g}=115 \mathrm{aF} / \mu \mathrm{m}^{2}$ is deduced from a separate Hall measurement. We observe that $\rho$ decreases monotonically as $|n|$ increases, however the sharpness of the dips at $n=0$ varies drastically from sample to sample.

In order to analyze the difference between samples quantitatively, we employ the semi-classical Drude model to estimate the mobility of samples, $\mu=(e n \rho)^{-1}$. Fig. 2 display $\mu$ as a function of $n$ obtained from Fig. 1(a). Although $\mu$ defined in this way diverges at $V_{g}=V_{\text {Dirac }}$ (the portion of curves represented by broken lines) due to the trivial reason that $n=0$ nominally at the Dirac point, the limiting value of the mobility, $\mu_{L}$, defined in the large density limit $\left(n \sim 4 \times 10^{12} \mathrm{~cm}^{-2}\right)$ serves as a useful way to characterize the sample quality. We have measured a total of 19 samples in this experiment where $\mu_{L}$ ranges $2,000-20,000 \mathrm{~cm}^{2} /$ Vsec. Quite generally, for samples of poorer quality, the Dirac points shift more from $V_{g}=0$, indicating a larger, unintentional charge doping of unknown origin [15]. The inset to Fig. 2 shows explicitly the scattering time $\tau$ of the samples shown in Fig. 1, with $\tau=\mu E_{F} /\left(e v_{F}^{2}\right)$ by definition. The corresponding mean free path of the samples can be obtained from $l=v_{F} \tau$, where the Fermi velocity $v_{F} \approx 10^{6} \mathrm{~m} / \mathrm{sec}[4]$. We note that the resulting mean free path for both electrons and holes strongly depends on the density $n$, ranging from 10$500 \mathrm{~nm}$ in the most of the samples and density ranges.

The quality of the samples can be further differentiated by comparing their behavior of carrier density dependent conductivity. Fig. 3 shows $\sigma=\rho^{-1}$ as a function of $V_{g}$. Evidently, the samples with poorer quality $\left(\mu_{L}<5,000 \mathrm{~cm}^{2} / \mathrm{Vsec}\right)$ show a very broad and smooth maximum in $\rho\left(V_{g}\right)$ near the Dirac point followed by a linear relationship of $\sigma\left(V_{g}\right)$ in the large density limit. On the other hand, when the mobility of a sample is larger than $\sim 10,000 \mathrm{~cm}^{2} / \mathrm{Vs}, \sigma\left(V_{g}\right)$ forms a cusp around the Dirac point followed by a sublinear increase both in the electron and hole regimes. Since the scattering time $\tau$ goes as $\tau \sim \sigma n^{-1 / 2}$, this difference in the density dependence of $\sigma$ in the two different mobility regimes strongly indicates that different scattering mechanisms might be dominating the high carrier density transport in samples from the two limiting groups. Recent numerical calculations based on Ref. 7, 14] indeed show that $\sigma(n)$ changes from a linear dependence to a sublinear dependence as the scattering mechanism changes to short range scattering, such as atomic defects in the lattice, from long range scattering, such as ionized impurity scattering.

Comparing our experimental data in a quantitative fashion with these theoretical calculations [14], we estimate that the charged impurity concentration varies in the range of $2-15 \times 10^{11} \mathrm{~cm}^{-2}$. Compared to recent Raman data on graphene samples, where the typical impurity density $n_{i}$ is estimated to be $n_{i} \sim 3 \times 10^{11} \mathrm{~cm}^{-2}$, this value appears to be quite reasonable [16]. We note that the relevant scattering times entering Raman scattering level broadening and dc conductivity are not necessarily identical, and typically the level broadening is larger than the transport broadening for the same impurity density. This explains why the Raman impurity density estimate falls toward the lower end of our transport estimate.

Simple theoretical arguments show that long ranged charged impurity scattering is much more effective for scattering of carriers than short-range scattering [7, 14]. For example, given the same impurity density $n_{i} \sim$ $5 \times 10^{11} \mathrm{~cm}^{-2}, l \gtrsim 1 \mu \mathrm{m}$ for short-range scatterers but $l \sim 50 \mathrm{~nm}$ for charged impurity scatterers. Since the size of our samples $L$ is on the order of several microns, transport can be considered to be in the diffusive regime. A 


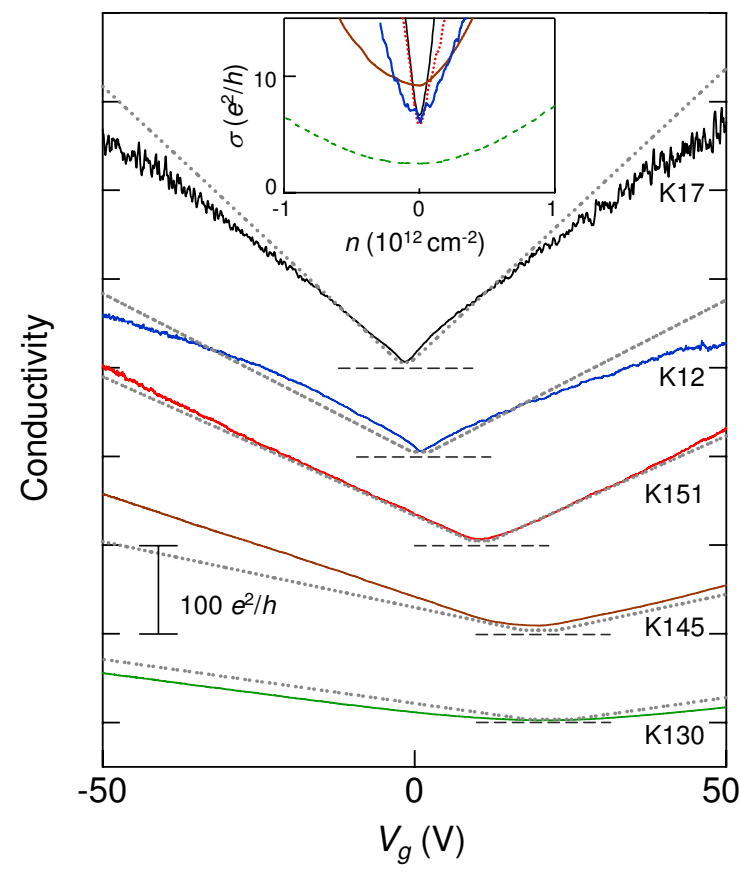

FIG. 3: (color online)The conductivity of five representative samples shown in Fig. 1 as a function of applied gate voltage (solid lines). For clarity, the each curves are displaced. The horizontal dashed lines indicate the zero conductance for each curves. Dotted curves are the corresponding theoretical results as explained in the text. The only fit parameter in the theory is the density of the random charged impurity centers in the substrate, which is taken to be (top to bottom in units of $10^{11} \mathrm{~cm}^{-2}$ ): $2.2,4.0,4.6,9.7,14.5$. The inset shows the detailed view of the density dependent conductivity near the Dirac point for the data in the main panel.

Boltzmann transport theory with charged impurity scattering can then be applied to solve for the conductivity self-consistently, and we obtain [17]

$$
\sigma\left(V_{g}\right) \approx\left\{\begin{array}{c}
C \frac{e^{2}}{h} \frac{n}{n_{i}} \text { for } n>n^{*} \\
C \frac{e^{2}}{h} \frac{n^{*}}{n_{i}} \text { for } n<n^{*}
\end{array}\right.
$$

where the carrier density is related to the gate voltage by $n=C_{g} V_{g} / e+\bar{n} ; n^{*}$ describes the self-consistent residual carrier density considering electron and hole puddle formation induced by the charged impurities; $\bar{n}$ is the induced carrier density in graphene by the charged impurity; and $C$ is a dimensionless numerical parameter describing the strength of scattering by the potential fluctuation considering full random phase approximation (RPA) screening. Considering the dielectric screening from the $\mathrm{SiO}_{2}$ substrate underneath the graphene samples, we estimate $C \approx 20$ [17. Within the RPA, the values of $n^{*}$ and $\bar{n}$ can be self-consistently determined by the charged impurity concentration $n_{i}$, and $d$, the distance between the graphene sheet and the charged impurity layer [17, 18].
We emphasize that in this model with a single free parameter $n_{i}$, Eq. 1 describes: (i) the linear tail mobility, (ii) the offset voltage $V_{\text {Dirac }}=-e \bar{n} / C_{g}$, (iii) the minimum conductivity given by $\approx 20 e^{2} / h\left(n^{*} / n_{i}\right)$ and (iv) the plateau width i.e. the range in gate voltage over which the conductivity saturates at its minimum value. Since these features are determined by the impurity concentration only, we now understand qualitatively that the behavior of conductivity of the cleaner samples (i.e. those with lower charged impurity density), in addition to having a higher slope, also have narrower minimum conductivity plateaus, smaller values of $V_{\text {Dirac }}$, and higher values of the minimum conductivity (i.e., $\sigma\left(V_{g}=V_{\text {Dirac }}\right)$ ). On the other hand, the dirtier samples have lower mobilities, wider plateau widths, and larger gate voltage offsets.

For a quantitative comparison, the theoretical model (Eq. 1) is now compared to the experimental data (Fig. 3). To avoid having too many fitting parameters in the theory, we just set $d \approx 1 \mathrm{~nm}$ (which is typical for $\mathrm{SiO}_{2}$ substrate [17]), keeping only $n_{i}$ as a single fitting parameter. This yields corresponding charged impurity concentrations for the samples in Fig. 3 ranging between $2-15 \times 10^{11} \mathrm{~cm}^{-2}$, where a reasonable agreement between experiment (solid curves) and theoretical curves can be seen. We note that that the some discrepancies between the experimental data and the simple model can be further understood: for example the sub-linear behavior seen in high quality samples (K17 and K12) is consistent with short-range scattering that becomes important only at very high density [14]. The electron and hole asymmetry often observed in poor quality samples (K130) can be explained by drift of charged impurity in the substrate. The large gate voltages used in the experiment $\left(V_{g} \sim 50 \mathrm{~V}\right)$ can shift the impurity trapped in the substrate, resulting in the adjustment of $d$ for electrons and holes. Had we introduced two additional fit parameters (one to parameterize the amount of short-range scattering and another one to parameterize $d$ the distance between the charged impurities and the graphene sheet) we would have obtained exact quantitative agreement. Given the reasonable agreement between theory and experiments for several different samples in Fig. 3, we do not see any advantages in obtaining an exact quantitative agreement by doing this additional data fitting.

We now address the minimum value of the conductivity, $\sigma_{\min }$ observed in our samples. Fig. 4 displays $\sigma_{\min }$ versus the high-density, limiting mobility value $\mu_{L}$ for all our samples. The measured values of $\sigma_{\min }$ lie in the range of $2-12 e^{2} / h$. Most samples have $\sigma_{\min }>4 e^{2} / h$, in contrast to an earlier study [2] reporting a clustering of $\sigma_{\min } \approx 4 e^{2} / h$ (horizontal dotted line). Several theories [5, 7] based on homogeneous graphene sheets predict a universal value of $4 e^{2} / \pi h$ for $\sigma_{\min }$ (horizontal dashed line), which is almost an order of magnitude lower than in most of our samples. The sample dependent $\sigma_{\min }$ was recently argued to arise from the inhomogeneous charge 


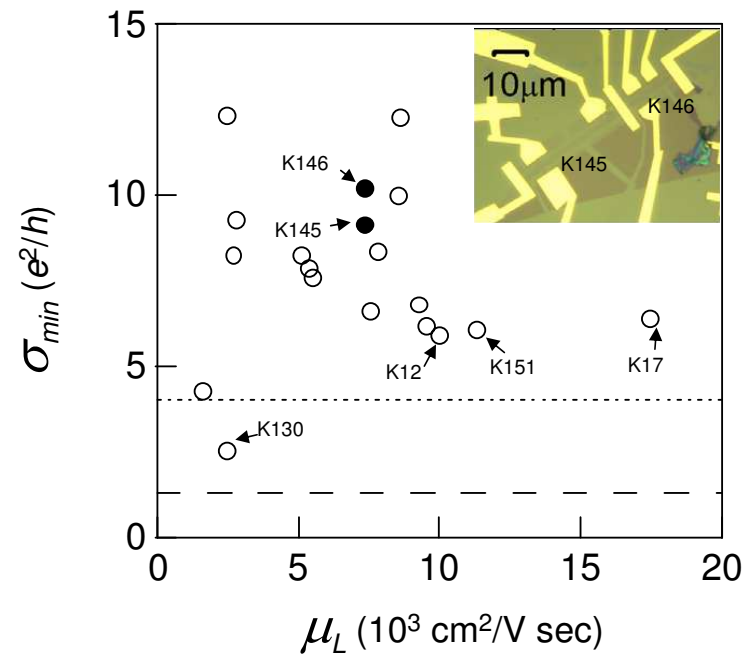

FIG. 4: The minimum conductivity and limiting mobility values at metallic regime for all 19 samples measured at $1.6 \mathrm{~K}$. Filled symbols indicate two devices, K145 and K146 made out of the same graphene flake as shown in the optical microscope image in the inset.

distribution near the Dirac point [4, 14]. More recently, theoretical work based on numerical calculation also indicates a non-invariant $\sigma_{\min }$ scaling with the system size in clean limit [19, 20]. Further careful consideration is needed for disordered samples. In the low density limit near the Dirac point where the carrier concentration becomes smaller than the charged impurity density, the system breaks up into puddles of electrons and holes where a duality in two dimensions guarantees that locally transport occurs either through the hole channel or the electron channel. Percolation resistance of these electron and hole puddles are the key to understanding the experimentally observed minimum conductivity [21]. The charged impurities induce a density distribution in the graphene sample where the presence of both electrons and holes carriers implies that both positive and negative fluctuations are screened by graphene [17]. It is the residual density given by $n^{*}$ that is responsible for the minimum conductivity, and therefore we expect that the value of the minimum conductivity will depend on the impurity concentration. Very recent scanning single electron transistor microscopy demonstrated such electronhole puddle formation near the charge neutral point in graphene [22].

In conclusion, we report zero-field transport properties of more than a dozen graphene samples with different mobility values. The minimum conductivity is found to be strongly sample dependent, yielding values an order of $e^{2} / h$ with a non-universal prefactor. Density inhomogeneities across the graphene sheet may be responsible for this large spread in conductivity.

We thank A. Geim, J. Yan, and I. Aleiner for helpful discussions. This work is supported by the DOE (DE-FG02-05ER46215 and DMR-03-52738), ONR (N000150610138), NSEC (CHE-06-41523 and DMR-03$52738)$, W. M. Keck foundation and the New York State Office of Science, Technology, and Academic Research (NYSTAR). The work at Maryland is supported by USONR, LPS-NSA, and Microsoft Q-Project.

$\dagger$ Current address: Dept. of Chemistry/Physics, University of California at Berkeley.

[1] K. S. Novoselov, A. K. Geim, S. V. Morozov, D. Jiang, Y. Zhang, S. V. Dubonos, I. V. Grigorieva, A. A. Firsov, Science 306, 666 (2004).

[2] K. S. Novoselov, A. K. Geim, S. V. Morozov, D. Jiang, M. I. Katsnelson, I. V. Grigorieva, S. V. Dubonos, A. A. Firsov, Nature 438, 197 (2005).

[3] Y. Zhang, Y. -W. Tan, H. L. Stormer, P. Kim, Nature 438, 201 (2005).

[4] A. K. Geim, K. S. Novoselov, Nature Mat. 6, 183 (2007).

[5] J. A. Nilsson, A. H. Castro Neto, F. Guinea, and N. M. R. Peres, Phys. Rev. Lett. 97, 266801 (2006).

[6] M. I. Katsnelson, Eur. Phys. J. B 51, 157-160 (2006).

[7] K. Nomura, and A. H. MacDonald, Phys. Rev. Lett. 98, 076602 (2007)

[8] H. Suzuura, and T. Ando, Phys. Rev. Lett. 89266603 (2002).

[9] D. V. Khveshchenko, Phys. Rev. Lett. 97036802 (2006).

[10] E. McCann, K. Kechedzhi, V. I. Fal'ko, H. Suzuura, T. Ando, B. L. Altshuler, Phys. Rev. Lett. 97146805 (2006).

[11] I. L. Aleiner, and K. B. Efetov, Phys. Rev. Lett. 97 236801 (2006)

[12] S. V. Morozov, K. S. Novoselov, M. I. Katsnelson, F. Schedin, L. A. Ponomarenko, D. Jiang, A. K. Geim, Phys. Rev. Lett. 97016801 (2006).

[13] K. S. Novoselov, D. Jiang, T. Booth, V. V. Khotkevich, S. M. Morozov, A. K. Geim, Proc. Natl. Acad. Sci. U.S.A. 102, 10451 (2005).

[14] E. H. Hwang, S. Adam, S. Das Sarma, Phys. Rev. Lett. 98186806 (2007);

[15] T. O. Wehling, K. S. Novoselov, S. V. Morozov, E. E. Vdovin, M. I. Katsnelson, A. K. Geim, A. I. Lichtenstein, arXiv:cond-mat: 0703390 (2007);

[16] J. Yan, Y. Zhang, P. Kim, and A. Pinczuk, Phys. Rev. Lett. 98166802 (2007).

[17] S. Adam, E. H. Hwang, V. M. Galitski, S. Das Sarma, arXiv:cond-mat: 0705.1540 (2007).

[18] V. M. Galitski, S. Adam and S. Das Sarma, arXiv:condmat: 0702117 (2007);

[19] J. H. Bardarson, J. Tworzydlo, P. W. Brouwer, and C. W. J. Beenakker, arXiv:cond-mat: 07050886 (2007).

[20] K. Nomura, M. Koshino, and S. Ryu, arXiv:0705.1607.

[21] V. V. Cheianov, V. I. Falko, B. L. Altshuler, I. L. Aleiner, arXiv:cond-mat: 07062968 (2007).

[22] J. Martin, N. Akerman, G. Ulbricht, T. Lohmann, J. H. Smet, K. von Klitzhing, A. Yacoby, arXiv:cond-mat: 07052180 (2007). 